\title{
Is a Molecular Adiabatic Approximation Appropriate to Positronic Atoms and Molecules?
}

\author{
J.R. Mohallem* \\ Laboratório de Átomos e Moléculas Especiais \\ Departamento de Física, ICEx, Universidade Federal de Minas Gerais \\ P.O. Box 702, 30123-970, Belo Horizonte, MG, Brasil \\ The adiabatic approximation to positronic atoms and molecules was \\ considered as an option to the computationally unfeasible methods that treat \\ all particles in a common footing, in two different approaches communicated \\ in the 37th PSPA. Here we present further assessment and comparison of \\ the two approaches as a way of evaluating the potential of adiabatic or, as \\ we found preferable, molecular approaches.
}

PACS numbers: 36.10.-k, 36.10.Dr, 78.70.Bj, 82.30.Gg

\section{Introduction}

Positronic atoms and molecules represent a special challenge to theoreticians, since the presence of a light but positively charged positron makes unfeasible the traditional approaches based on the different masses of the constituent particles. The solution seemed to be to treat all particles on a common footing in full nonadiabatic quantum mechanical calculations. From this very starting point the possibility of interpreting the physical and chemical properties of positronic systems in terms of a common basic model is lost; each system is a particular problem. However, the prospect of predicting bound and resonant states not yet observed as well as to calculate scattering states to compare theoretical and experimental cross-sections boosted the theoretical research on positronic systems with these methods, mainly in the last decade. Three main all-body methods are worth to be cited. The powerful stochastic variational method (SVM) [1, 2] and quantum Monte Carlo (QMC) [3-5] generated converged binding energies and annihilation rate constants ${ }^{\dagger}$ for small positronic atoms. However, they seem to have

\footnotetext{
*e-mail: rachid@fisica.ufmg.br

${ }^{\dagger}$ During the meeting it was suggested by Prof. G. Duplâtre the use of the term "annihilation rate constant" instead of just "annihilation rate" since this last quantity is time dependent.
} 
reached their computational limit. Any reliable application to few-active-electrons positronic atom or molecule is predicted to take even years of computing time. Applications to positronic molecules have been considered mainly on the basis of the configuration interaction (CI) method [6, 7], in which electron (through a determinantal wave function) and positron orbital excitations are allowed. Nevertheless, small truncated CI expansions and frozen molecular geometries generate doubtful results [8-10], while larger expansions and allowance of geometry relaxation turns it to be computationally limited, too. Furthermore, it behaves poorly in the calculation of annihilation rate constants. As a result of these difficulties, this research suffered a depression in the last years, with the number of published results being strongly diminished.

In face of this dilemma, a real change of paradigm is being considered: the idea of treating the positron as a pseudonucleus has eventually appeared in the literature, mostly in phenomenological or model calculations [11-16]. It has been proposed to go further and to perform a widespread adiabatic separation of motions of positively charged particles, positron plus nuclei, and electrons. The answer to the title question has not been given yet, but some insight provided by the discussions during the 37th PSPA as well as some new motivations can be advanced here.

Besides the vital consideration of computational time, at least one further previous motivation for such an approach can be devised. It is well known that molecular adiabatic methods describe satisfactorily the electronic density on the nuclei, since electronic basis functions can be, and commonly are, centered on the nuclei. The perspective of repeating this feature for the positron as a special nucleus is a very stimulating one, since it predicts a good performance of an adiabatic based method for annihilation rate constants. Two methodologies has been communicated in the 37th PSPA, both in early phases of development, but some of their features can be already assessed and compared. In the following analysis, we consider the interaction of a positron with a single atom, just for simplicity.

\section{Models}

In any adiabatic approximation the total Hamiltonian is partitioned in others involving different degrees of freedom. The Polish (Pol) initiative is based on a partition of the clamped-nuclei Hamiltonian in an electronic one and a positronic one following the interesting idea of taking the positronic charge not as +1 (in atomic units, a.u.) but as effective charges $q_{\mathrm{p}}$, that appears in the electronic Hamiltonian and $\left(1-q_{\mathrm{p}}\right)$, that appear in the positronic one [17]. The two Hamiltonians are defined accordingly, the electronic equation is solved generating an "electronic energy" that, together with the effective electrostatic repulsion and the common adiabatic correction form the potential energy curves (PECs) for positron motion. Positronic states are obtained from the solution of the positronic equation with these PECs. The adiabatic correction accounts for the small mass of the positron. 
On the other hand, in the Brazilian (Braz) approach the positron is taken in the equations as a real nucleus but its finite mass correction is inserted in the electronic Hamiltonian through a special and approximate transformation from the laboratory-fixed to the system-fixed reference frames [18]. This makes the electronic Hamiltonian dependent on the nuclear masses. This electronic Hamiltonian generates the electronic states having the signature of the nuclear masses, particularly the small positron mass. Electronic energies plus positron-nucleus repulsion yields the PECs and solution of the resulting "nuclear" equation gives the positronic states.

Thus, both methods aim at describing better the electronic density on the positron by allowing electronic basis functions to be positron-centered (though the Pol method allows the Gaussian functions to flow in order to be more variational), but are strongly different in spirit.

Let us now compare them in a case they have both been applied to, the positronium hydride, HPs. This case has the advantage that HPs is recognized as an actual nonadiabatic system, in view of the highly correlated motion of the electron-positron pair in the positronium $(\mathrm{Ps})$ cluster. The Pol model is more sophisticated on its adiabatic single level due to its charge partition effect. As a consequence, it yields a better eigenvalue, -0.7762 a.u. [17], than the purely adiabatic Braz one, -0.7668 a.u. [15], both to be compared to the exact eigenvalue -0.7891 a.u. [19]. This improvement should be connected to the variational flexibility allowed by the introduction of the $q_{\mathrm{p}}$ parameter, reflected on the unphysical peaks in the PECs (see Fig. 1 in [17]). However, in view of the exact value of the threshold energy $(\mathrm{H}+\mathrm{Ps})$ yielded by both methods, the calculated binding energies are quite poor so that the application is reported by the Pol authors as a failure. On the other hand, both methods give good values for the annihilation rate constant (Braz), or for the equivalent electron-positron coalescence (Pol), accordingly to the very motivation of introducing such methods in the field. Unfortunately, there is no common application of the two methods to a positron-neutral atom system, for which the binding energy is orders of magnitude lower. Anyway, in such a case both adiabatic eigenvalues and threshold energy become approximate, at least partial error compensation would happen so that the Pol approach is supposed to still work reasonably, as suggested by the model applications [17].

Except for tightly bonded Ps complexes, the Braz approach does not generate bound states on the adiabatic level at all, though the resulting PECs describe properly the threshold behavior and the electron densities for fixed positron positions [20]. This level has been considered, on the other hand, as the first step in a two-step molecular approach to positronic systems. This first step applies just to yield the electronic states and the PECs needed to proceed toward the final calculations. It generates a set of electronic states whose nonadiabatic couplings are needed to describe any positronic system exactly. To illustrate the second step 
we resort again to HPs. Instead of solving complicated coupled equations, it is possible to mimic the main nonadiabatic effect with a phenomenological procedure suggested by physical analysis. The ground state PEC is well isolated, meaning that no strong local coupling (crossing or pseudo-crossing) takes place. The whole effect of coupling excited states to the ground one would be to allow the excitation of a electron so that it becomes able to follow the positron motion, forming the Ps cluster. This effect can be simulated by just keeping the motion of the positron in the ground state PEC but changing its mass to an effective one accounting also to the mass of an electron it draws in its motion. With this empirical procedure, the almost exact energy eigenvalue -0.7879 a.u. is generated [15].

Let us proceed now to a general analysis. The energy eigenvalues of the nonadiabatic HPs given by the two adiabatic methods are poor in the sense of presenting errors of $2.8 \%$ error for Braz and $1.3 \%$ for Pol relative to the exact eigenvalue. Although not valuable to calculate the binding energies, they are quite close to the exact eigenvalue, meaning that the adiabatic component of the full HPs wave function is very large and accounts for some properties other than the binding energy, as annihilation rate constants. The "nonadiabaticity" of positron complexes seems to be just a small step over the adiabatic one. This means that once an adiabatic step takes place, we become quite closer to the actual solution of the problem. This starting point is not to be underestimated. The step-up is easier in the Braz approach, in consequence of its resemblance with the well known adiabatic and nonadiabatic procedures within the Born-Huang theory of molecules [21]. This does not mean, however, that an equivalent step cannot be found for the Pol approach.

\section{Conclusions}

In conclusion, although further comparison cannot be made at this point, it seems that Pol is a very appropriate approach for systems that behave adiabatically, with a probable better performance than Braz. On the other hand, the nonadiabatic step of the Braz approach seems to admit to reach equivalent accuracy of the more sophisticated methods cited in the indroduction. All these considerations justify our confidence that improved adiabatic (or preferred molecular) based approaches can be developed in order to increase our predictive capacity for positronic systems in the future.

\section{Acknowledgments}

The author profited of useful discussions with Dr. K. Strasburger during the meeting. Attendance to 37th PSPA has been supported by Fapemig and $\mathrm{CNPq}$ (Brazilian agencies) through grants and by the Organizers, to whom special acknowledgements are due. 


\section{References}

[1] Y. Suzuki, K. Varga, Stochastic Variational Approach to Quantum-Mechanical Few-Body Problems, Springer, New York 1998.

[2] G. Ryzhikh, J. Mitroy, K. Varga, J. Phys. B, At. Mol. Opt. Phys. 31, 3965 (1998).

[3] D. Bressanini, P.J. Reynolds, Adv. Chem. Phys. 105, 37 (1998).

[4] D. Bressanini, M. Mella, G. Morosi, J. Chem. Phys. 108, 4756 (1998).

[5] D.M. Schrader, T. Yoshida, K. Iguchi, Phys. Rev. Lett. 68, 3281 (1992).

[6] O.G. Ludwig, R.G. Parr, Theor. Chim. Acta 5, 440 (1966).

[7] K. Strasburger, H. Chojnacki, Chem. Phys. Lett. 241, 485 (1995).

[8] M. Tachikawa, R.J. Buenker, M. Kimura, J. Chem. Phys. 119, 5005 (2003).

[9] J.R. Mohallem, C.P. Gonçalves, J. Chem. Phys. 121, 5553 (2004).

[10] M. Tachikawa, R.J. Buenker, M. Kimura, J. Chem. Phys. 121, 9191 (2004).

[11] M.W. Karl, H. Nakanishi, D.M. Schrader, Phys. Rev. A 30, 1624 (1984).

[12] J.R. Mohallem, J. Phys. B, At. Mol. Opt. Phys. 32, 3805 (1999).

[13] J.R. Mohallem, F. Rolim, C.P. Gonçalves, Mol. Phys. 99, 87 (2001).

[14] F. Rolim, J.P. Braga, J.R. Mohallem, Chem. Phys. Lett. 332, 139 (2000).

[15] F. Rolim, T. Moreira, J.R. Mohallem, Braz. J. Phys. 34, 1197 (2004).

[16] H. Stachowiak, E. Boronski, Phys. Rev. B 71, 245107 (2005).

[17] K. Strasburger, M. Wolcyrz, Mol. Phys. 105, 467 (2007).

[18] J.R. Mohallem, F. Rolim, C.P. Gonçalves, J. Phys. B, At. Mol. Opt. Phys. 37, 1045 (2004).

[19] M. Mella, G. Morosi, D. Bressanini, J. Chem. Phys. 111, 108 (1999).

[20] D. Assafrão, H.R. James Walters, J.R. Mohallem, Nucl. Instrum. Methods Phys. Res. B 266, 491 (2008).

[21] M. Born, K. Huang, Dynamical Theory of Crystal Lattices, Oxford University Press, New York 1956. 